Editorial

\title{
Lamina cribrosa measurements for glaucomatous eyes: a new focus point in optical coherence tomography
}

Keywords: lamina cribrosa, measurements, optical coherence tomography, glaucoma, anterior surface depth, curvature index, laminar thickness, prelaminar tissue thickness

Abbreviations: LC, lamina cribrosa; RGC, retinal ganglion cell; IOP, intraocular pressure; GON, glaucomatous optic neuropathy; RNFL, retinal nerve fiber layer; OCT, optical coherence tomography; EDI-OCT, enhanced imaging OCT; SS-OCT, swept source OCT; ALCS, anterior LC surface; ALCSD, anterior LC surface depth; PTT, prelaminar tissue thickness; LCT, LC thickness; BM, Bruch's membrane; BMO, BM opening; LCCI, LC curvature index; LCCD, LC curve depth

\section{Editorial}

Lamina cribrosa (LC) is the part of the sclera with a mesh-like structure and it is the exit zone from the globe of retinal ganglion cell (RGC) axons. It has a lot of pores formed by the multi-layered network of collagen fibers. It has been considered that LC maintains the pressure gradient between intraocular pressure (IOP) and the periocular pressure and it structurally supports RGC axons. As LC is structurally weaker than other parts of sclera which are thicker and denser, it is highly sensitive to the effects of the IOP elevation..$^{1,2}$ $\mathrm{LC}$ is a dynamic structure responding with thinning or thickening to IOP changes. Following sudden IOP increase, optic cup excavation associates with thinning in prelaminary tissue. The IOP elevation causes the compression, posterior displacement or bulging of the LC, deformation of the pores, kinking and pinching of the RGC axons and blood vessels traversing through the laminar pores. It has been thought that the blockade of axoplasmic flow within RGC or ischemic damage to RGC axons due to compressional LC deformation caused by the distortion or posterior displacement of the LC might lead to glaucomatous optic neuropathy (GON). Additionally, it has been demonstrated in experimental studies that posterior displacement of LC precedes early surface-detected structural damage and retinal nerve fiber layer (RNFL) loss. Thus, LC is the putative primary region of the axonal injury in RGC in GON. ${ }^{1,2}$

Enhanced depth imaging optical coherence tomography (EDIOCT) and swept source OCT (SS-OCT) have provided better visualization of the vitreoretinal interface, retina, optic disc, choroid and choroidoscleral junction. In recent years, these imaging modalities have been also used in the imaging of LC in especially patients with glaucoma and healthy subjects, and the posterior displacement and focal defects in LC detected by OCT have been linked to GON. ${ }^{3-10}$ Additionally, it has been also reported that the LC moved anteriorly with the reduction in the depth of LC following IOP lowering in the patients with glaucoma. The reversibility of the LC has been related to both the anterior displacement of the $\mathrm{LC}$ and the thickening of the prelaminary tissue. ${ }^{11-13}$ Moreover, it has been demonstrated in
Volume 8 Issue I - 2018

\author{
Burak Turgut \\ Department of Ophthalmology, Yuksek Ihtisas University, Turkey
}

\author{
Correspondence: Burak Turgut, Professor of Ophthalmology, \\ Yuksek Ihtisas University, Faculty of Medicine, \\ Department of Ophthalmology, 06520, Ankara, Turkey, Tel +90 \\ 3122803601 , Fax +90 3122803605, \\ Email burakturgut@yiu.edu.tr
}

Received: February 21, 2018 | Published: February 23, 2018

experimental glaucoma that the displacement of the LC might precede structural LC damages and early RNFL defects. ${ }^{14}$ The measurement of the parameters related with LC such as anterior LC surface (ALCS) depth, prelaminar tissue thickness (PTT) and LC thickness (LCT) may reveal posterior LC displacement and the thinning of LC (Figure 1). ${ }^{15-}$ ${ }^{21}$ ALCS depth (ALCSD) is defined as the distance from the Bruch's membrane opening (BMO) level to the ALCS. BMO is defined as the termination point of Bruch's membrane (BM) and it seemsas a highreflection and high-contrast line in EDI-OCT. ALCS is the surface between the prelaminar tissue and the lamina cribrosa. Recent reports suggest that ALCSD might vary according to sex and axial length and it should be measured at various planes and sections. ${ }^{7,8,11,12,15-17}$

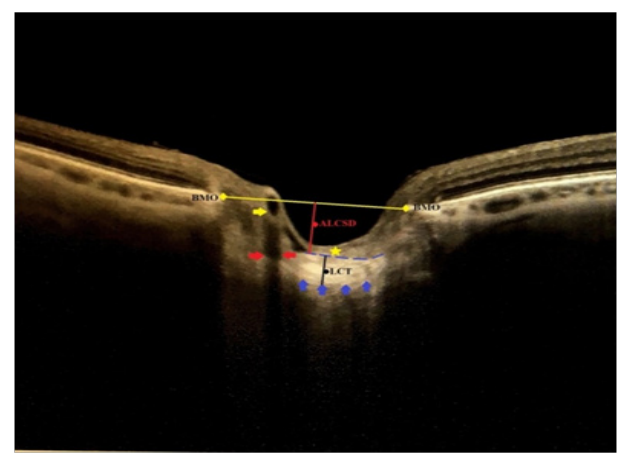

Figure I Some terms and parameters to be used in lamina cribrosa morphology in glaucoma. BMO, bruch membrane opening; ALCSD, anterior lamina cribrosa surface depth; LCT, lamina cribrosa thickness. Yellow and red arrows indicate the vascular trunk in the optic disc and its shadowing, respectively. Yellow star points out the prelaminary tissue. Break blue line indicates anterior lamina cribrosa surface. Blue arrows point out the possible posterior lamina cribrosa surface. 
LCT is defined as the distance between the anterior and posterior borders of the hiper-reflective region visible beneath the ONH in a crosssectional B-scan SS-OCT or EDI-OCT image. It has been reported that LCT was thinner in eyes with primary open angle glaucoma and normal tension glaucoma compared with normal eyes. ${ }^{11-13}$ However, the clear detection of the posterior border of the LC may always not be possible in OCT and its exact borders may be only confirmed histologically. ${ }^{18}$ The prelaminar tissue is composed primarily of RGC axons and glial cells which support them. PTT is measured as the distance between ALCS and vitreous-optic nerve head interface or anterior prelaminar surface in the region enclosed by the Bruch's membrane opening. ${ }^{11,13,19}$ In a recent study, it has been demonstrated that prelaminar tissue in healthy eyes had significantly thicker than the eyes with glaucoma or glaucoma suspect, and it was significantly thicker in the eyes with glaucoma suspect than glaucomatous eyes. Additionally, it has been reported that of the anterior LC boundary was significantly more visible in glaucomatous eyes compared to healthy eyes. ${ }^{19}$ Lamina cribrosa curvature index (LCCI) is an LCrelated index defined by Lee et al to quantify the posterior bowing of the LC using with SS-OCT. ${ }^{7}$ LCCI is calculated by dividing the lamina cribrosa curve depth (LCCD) by the width (W) of the ALCS reference line and multiplying by 100 (LCCI $=(\mathrm{LCCD} / \mathrm{W}) \mathrm{X} 100)$. $\mathrm{LCCD}$ is determined as the maximum depth from this reference line was set in each B scan by connecting the two points on the ALCS that met with the lines drawn from each Bruch's membrane termination point perpendicularly to the BMO reference line to the ALCS. "W", width, is the length of this reference line. It has been reported by Lee et al that LCCI has discriminating capability between glaucoma and healthy eyes. ${ }^{7}$

\section{Conclusion}

All of these LC related measurements or calculations may be beneficial in the diagnosis and the evaluation of treatment and prognosis in glaucoma patients. However, it should not forget that above-mentioned measurements especially, the measurement of LCT and especially, the visualization of posterior LC surface might be affected by OCT signal ratio, individual variations in PTT, optic disc pit, focal LC defects, neuroretinal rim, thick prelaminary tissue, shadowing due to vascular trunk in optic disc and LC segmentation errors. $^{7-10,15-21}$ Further studies on LC morphology and indices will be able to provide more significant knowledge for the usage of LC parameters for early diagnosis and follow-up in glaucomatous eyes.

\section{Acknowledgments}

None.

\section{Conflicts of interest}

The author declares that there is no conflict of interest regarding the publication of this paper.

\section{Funding}

The author received no financial support for the research, authorship, and/or publication of this article.

\section{Authorship contributions}

Concept and Design, Data Collection, Literature Search, Writing: Analysis, Interpretation: Burak Turgut.

\section{References}

1. Quigley HA, Addicks EM, Green WR, et al. Optic nerve damage in human glaucoma. II. The site of injury and susceptibility to damage. Arch Ophthalmol. 1981;99(4):635-649.

2. Seo JH, Kim TW, Weinreb RN. Lamina cribrosa depth in healthy eyes. Invest Ophthalmol Vis Sci. 2014;55(3):124-1251.

3. Kim YW, Jeoung JW, Kim YK, et al. Clinical implications of in vivo lamina cribrosa imaging in glaucoma. J Glaucoma. 2017;26(9):753-761.

4. Kim TW, Kagemann L, Girard MJ, et al. Imaging of the lamina cribrosa in glaucoma: perspectives of pathogenesis and clinical applications. Curr Eye Res. 2013;38(9):903-909.

5. Rebolleda G, Muñoz Negrete FJ. Enhanced Depth Imaging- optical coherence tomography technique and the lamina cribrosa in glaucoma. Arch Soc Esp Oftalmol. 2014;89(4):133-135.

6. Sigal IA, Wang B, Strouthidis NG, et al. Recent advances in OCT imaging of the lamina cribrosa. Br J Ophthalmol. 2014;98 Supp1 2:ii34-ii39.

7. Lee SH, Kim TW, Lee EJ, et al. Diagnostic power of lamina cribrosa depth and curvature in glaucoma. Invest Ophthalmol Vis Sci. 2017;58(2):755762.

8. Tan NY, Koh V, Girard MJ, et al. Imaging of the lamina cribrosa and its role in glaucoma: a review. Clin Exp Ophthalmol. 2017;doi:10.1111/ ceo.13126.

9. Downs JC, Girkin CA. Lamina cribrosa in glaucoma. Curr Opin Ophthalmol. 2017;28(2):113-119.

10. Abe RY, Gracitelli CPB, Diniz-Filho A, et al. Lamina Cribrosa in Glaucoma: Diagnosis and Monitoring. Curr Ophthalmol Rep. 2015;3(2):74-84.

11. Agoumi Y, Sharpe GP, Hutchison DM, et al. Laminar and prelaminar tissue displacement during intraocular pressure elevation in glaucoma patients and healthy controls. Ophthalmology. 2011:118(1):52-59.

12. Tun TA, Thakku SG, Png O, et al. Shape changes of the anterior lamina cribrosa in normal, ocular hypertensive, and glaucomatous eyes following acute intraocular pressure elevation. Invest Ophthalmol Vis Sci. 2016;57(11):4869-4877.

13. Barrancos C, Rebolleda G, Oblanca N, et al. Changes in lamina cribrosa and prelaminar tissue after deep sclerectomy. Eye. 2014;28(1):58-65.

14. Kim JA, Kim TW, Weinreb RN, et al. Lamina cribrosa morphology predicts progressive retinal nerve fiber layer loss in eyes with suspected glaucoma. Sci Rep. 2018;8(1):738.

15. Park SC, Brumm J, Furlanetto RL, et al. Lamina cribrosa depth in different stages of glaucoma. Invest Ophthalmol Vis Sci. 2015;56(3):2059-2064.

16. Kim M, Bojikian KD, Slabaugh MA, et al. Lamina depth and thickness correlate with glaucoma severity. Indian J Ophthalmol. 2016;64(5):358363.

17. Oh BL, Lee EJ, Kim H, et al. Anterior lamina cribrosa surface depth in open-angle glaucoma: relationship with the position of the central retinal vessel trunk. PLoS One. 2016;11(6):e0158443.

18. Strouthidis NG, Grimm J, Williams GA, et al. A comparison of optic nerve head morphology viewed by spectral domain optical coherence tomography and by serial histology. Invest Ophthalmol Vis Sci 2010;51(3):1464-1474

19. Lucy KA, Wang B, Schuman JS, et al. Thick prelaminar tissue decreases lamina cribrosa visibility. Invest Ophthalmol Vis Sci. 2017;58(3):17511757. 
20. Kwun Y, Han JC, Kee C. Comparison of lamina cribrosa thickness in normal tension glaucoma patients with unilateral visual field defect. Am J Ophthalmol. 2015;159(3):512-518.
21. Chung HS, Sung KR, Lee JY, et al. Lamina cribrosa-related parameters assessed by optical coherence tomography for prediction of future glaucoma progression. Curr Eye Res. 2016;41(6):806-813. 